EXTENDED REPORT

\title{
Comparative efficacy of pharmacological and non-pharmacological interventions in fibromyalgia syndrome: network meta-analysis
}

\author{
Eveline Nüesch, ${ }^{1,2}$ Winfried Häuser, ${ }^{3,4}$ Kathrin Bernardy, ${ }^{5,6}$ Jürgen Barth, ${ }^{1}$ Peter Jüni ${ }^{1}$
}

- Additional data are published online only. To view the files please visit the journal online (http://dx.doi.org/ 10.1136/annrheumdis-2011201249).

\section{${ }^{1}$ Institute of Social and}

Preventive Medicine (ISPM),

University of Bern, and CTU

Bern, Bern University Hospital,

Bern, Switzerland

${ }^{2}$ Faculty of Epidemiology and

Population Health, London

School of Hygiene and Tropical

Medicine, University of

London, London, UK

${ }^{3}$ Department of Internal

Medicine 1, Klinikum

Saarbrücken, Saarbrücken,

Germany

${ }^{4}$ Department of Psychosomatic

Medicine and Psychotherapy,

Technische Universität

München, München, Germany

${ }^{5}$ Department of Internal

Medicine, Complementary and Integrative Medicine,

University of Duisburg-Essen,

Kliniken Essen-Mitte, Essen,

Germany

${ }^{6}$ Department of Pain

Management,

Berufsgenossenschaftliches

Universitätsklinikum

Bergmannsheil, Ruhr University

Bochum, Bochum, Germany

\section{Correspondence to}

Dr Eveline Nüesch, Division of Clinical Epidemiology and

Biostatistics, Institute of Social and Preventive Medicine (ISPM), University of Bern, Bern 3012, Switzerland; enueesch@ispm.unibe.ch

Received 25 December 2011 Accepted 31 May 2012

\begin{abstract}
Objectives To synthesise the available evidence on pharmacological and non-pharmacological interventions recommended for fibromyalgia syndrome (FMS).

Methods Electronic databases including MEDLINE, PsycINFO, Scopus, the Cochrane Controlled Trials Registry and the Cochrane Library were searched for randomised controlled trials comparing any therapeutic approach as recommended in FMS guidelines (except complementary and alternative medicine) with control interventions in patients with FMS. Primary outcomes were pain and quality of life. Data extraction was done using standardised forms.
\end{abstract}

Results 102 trials in 14982 patients and eight active interventions (tricyclic antidepressants, selective serotonin reuptake inhibitors, serotonin noradrenaline reuptake inhibitors (SNRIs), the gamma-amino butyric acid analogue pregabalin, aerobic exercise, balneotherapy, cognitive behavioural therapy (CBT), multicomponent therapy) were included. Most of the trials were small and hampered by methodological quality, introducing heterogeneity and inconsistency in the network. When restricted to large trials with $\geq 100$ patients per group, heterogeneity was low and benefits for SNRIs and pregabalin compared with placebo were statistically significant, but small and not clinically relevant. For nonpharmacological interventions, only one large trial of CBT was available. In medium-sized trials with $\geq 50$ patients per group, multicomponent therapy showed small to moderate benefits over placebo, followed by aerobic exercise and CBT.

Conclusions Benefits of pharmacological treatments in FMS are of questionable clinical relevance and evidence for benefits of non-pharmacological interventions is limited. A combination of pregabalin or SNRIs as pharmacological interventions and multicomponent therapy, aerobic exercise and CBT as non-pharmacological interventions seems most promising for the management of FMS.

\section{INTRODUCTION}

Key symptoms of fibromyalgia syndrome (FMS) are chronic widespread pain associated with cognitive dysfunction, sleep disturbances and physical fatigue. ${ }^{2}$ Patients often report high levels of disability and poor quality of life, and an extensive use of medical care. ${ }^{3}$ In the absence of suitable laboratory tests, diagnosis is established by a history of key symptoms and the exclusion of somatic diseases sufficiently explaining these symptoms. ${ }^{2} 4$ The estimated overall prevalence of FMS is $2.9 \%$ in the general population of five European countries. ${ }^{5}$ The definite aetiology of FMS remains unknown. ${ }^{4}$ Since specific treatment aimed at altering the pathogenesis is not possible, the therapeutic focus is on symptom reduction.

Systematic reviews and evidence-based guidelines provide healthcare professionals and patients with a guide through the large variety of pharmacological and non-pharmacological treatment options offered to and used by patients with FMS. ${ }^{6}$ The American Pain Society ${ }^{7}$ and the Association of Scientific Medical Societies in Germany ${ }^{4}$ strongly recommend a pharmacological intervention (amitriptyline) and several non-pharmacological treatments (aerobic exercise, cognitive behavioural therapy (CBT), multicomponent therapy). Conversely, the European League Against Rheumatism (EULAR) have given only a strong recommendation for a variety of pharmacological therapies (eg, tricyclic antidepressants (TCAs), serotonin-noradrenaline reuptake inhibitors (SNRIs), serotonin reuptake inhibitors (SSRIs), gamma-amino butyric acid analogues (GABA) such as pregabalin) but weak recommendations for non-pharmacological therapies such as aerobic exercise, CBT and multicomponent therapy. ${ }^{8}$ Recommendations for first-line treatment options of FMS, however, are hampered by the lack of head-to-head comparisons of pharmacological versus non-pharmacological treatments.

Network meta-analyses allow a unified coherent analysis of all randomised controlled trials comparing pharmacological and non-pharmacological treatments head-to-head or with a control intervention, while fully respecting randomisation..$^{9-11}$ We performed a systematic review with network meta-analysis of randomised trials in patients with FMS evaluating effects of pharmacological and non-pharmacological interventions recommended in FMS guidelines on pain and quality of life. We provide an overall synthesis of available data that can be used to guide treatment decisions and examined the potential for bias due to methodological flaws or small-study effects. ${ }^{12-15}$

\section{METHODS}

\section{Literature search and trial selection}

We searched MEDLINE, PsycINFO, Scopus, the Cochrane Controlled Trials Registry and the Cochrane Library, all from inception through 31 December 2011. The search strategy has been previously described. ${ }^{16-21}$ We included treatment 
options approved by the Food and Drug Administration for FMS or recommended by evidence-based guidelines on the management of $\mathrm{FMS}^{4}$ antidepressants, GABA-analogues, aerobic exercise, balneotherapy, CBT and multicomponent therapies (at least one exercise component and at least one psychological component). Therapeutic interventions were excluded if they had been studied in fewer than four trials or were classified as alternative and complementary medicine according to National Institutes of Health (NIH) criteria (http://nccam.nih. gov/health/whatiscam/). We included randomised controlled trials in patients with FMS defined according to established criteria, ${ }^{22-24}$ comparing any of these pre-specified therapeutic approaches with control interventions. Waiting list or treatment as usual were classified as non-intervention control, drug placebo or sham intervention as placebo control and interventions deemed a priori to be only minimally active (such as relaxation, education or passive physical therapy) as minimally active control. No language restrictions were applied. Two reviewers independently evaluated reports for eligibility and contacted a third reviewer to resolve disagreements.

\section{Outcomes}

Pain and quality of life were pre-specified as primary outcomes whereas the remaining key domains of FMS, ${ }^{25}$ sleep and fatigue were specified as secondary outcomes. Drop-outs, defined as patients who terminated the trial early for any reason during the treatment period, were extracted as a measure of acceptability. ${ }^{11}$ When there was more than one measure for a specific outcome, we gave preference to measures recommended by OMERACT. ${ }^{25}$

\section{Data extraction}

We used standardised forms of previous reviews to extract data on publication status, trial design, patients, characteristics and treatment regimens ${ }^{16-19}$ and to assess components of methodological trial quality (generation of randomisation sequence, allocation concealment, blinding of patients and handling of incomplete outcome data; for definitions see Methods S1 in the online supplement) ${ }^{12} 26$ independently by two reviewers. Results of the outcomes at the end of the treatment period were extracted by one reviewer (WH) and cross-checked by another (EN). We contacted corresponding authors of trials with incomplete outcome data. When necessary, means and measures of dispersion were approximated from figures in the reports.

\section{Data synthesis}

Whenever available we used results from intention-to-treat analyses. We expressed treatment effects as standardised mean differences (SMD) by dividing the difference in mean values between treatment groups at the end of treatment by the pooled standard deviation. If information for the calculation of pooled standard deviations was unavailable or ambiguous in a trial, we used the median pooled standard deviation from other trials using the same scale. Negative SMDs indicate a beneficial effect of the experimental intervention throughout. An SMD of -0.20 indicates small differences between groups whereas -0.50 suggests moderate and -0.80 large differences. ${ }^{27}$ Treatment effects for acceptability were expressed as risk ratios of dropouts.

Details of the statistical methods used for meta-analysis are provided in Methods S2 and S3 in the online supplement. In short, we used Bayesian hierarchical random effects models which fully preserve randomised treatment comparisons within trials. $^{10} 2829$ Pooled SMDs or risk ratios comparing different active and control interventions were estimated from the median of the posterior distribution, corresponding $95 \%$ credibility intervals $(95 \% \mathrm{CrI})$ from the 2.5 th and 97.5 th percentiles. Credibility intervals can be interpreted similarly to conventional CI. We examined heterogeneity between trials, goodness of model fit and inconsistency between direct and indirect estimates ${ }^{30} 31$ and performed conventional Bayesian random effects meta-analyses for all available direct comparisons. ${ }^{32}$

For primary outcomes we drew funnel plots of trials comparing any active intervention with non-intervention or placebo controls, ${ }^{33}$ enhanced by contours dividing the plots into areas of significance and non-significance at a two-sided $p=0.05,{ }^{34} 35$ and added lines of predicted treatment effects derived from univariable random effects meta-regression models using the standard error as explanatory variable. ${ }^{36} 37$ Funnel plot asymmetry was assessed with Egger's test. ${ }^{38}$

We stratified analyses according to adequate generation of random sequence, concealment of allocation, blinding, intention-to-treat analysis, sample size and exclusion of patients with inflammatory rheumatic disorders or mental disorders and derived $p$ values for interaction between trial characteristics and treatment effect as previously described. ${ }^{39} 40 \mathrm{We}$ pre-specified a cut-off of an average of 100 randomised patients per treatment group to distinguish between small and large trials. ${ }^{14}$ Since no large trials were available for most of the interventions and there was evidence for small-study effects, we explored the impact of trial size by a stepwise restriction of the analysis to trials of increasing sample size, with cut-offs of 25, 50 and 100 patients per treatment arm; $2 \times 25$ patients will yield more than $80 \%$ power to detect a large $\mathrm{SMD}$ of -0.80 , $2 \times 50$ patients will yield more than $80 \%$ power to detect a moderate to large SMD of -0.60 , and $2 \times 100$ patients more than $80 \%$ power to detect a small to moderate SMD of -0.40 at a two-sided $\alpha=0.05$. Networks of pharmacological and nonpharmacological treatments failed to connect at cut-offs of $2 \times 50$ and $2 \times 100$ patients. We therefore used differences between non-intervention control and placebo observed in overall analyses to connect the two networks through combination of placebo and non-intervention control groups.

\section{RESULTS}

\section{Selection and characteristics of included trials}

Figure 1 shows the flowchart for the selection of randomised trials in FMS. One hundred and two trials with 14982 patients were eligible and contributed to the network meta-analysis (table 1 and supplementary online table S1). The trials included a median of 69 randomised patients (range 14-1196) and were published between 1986 and 2011. Fifteen trials evaluated TCAs (1026 patients), 10 trials SSRIs (644 patients), 10 trials SNRIs (5980 patients) and 4 trials the GABA analogue pregabalin (2625 patients). Thirty-three trials evaluated aerobic exercise (2266 patients), 9 trials balneotherapy (387 patients), 20 trials CBT (1712 patients) and 15 trials multicomponent therapy (1751 patients). Thirty-eight trials had a non-intervention control (2689 patients), 40 trials had a placebo control (10 545 patients) and 23 trials had a minimally active control (2129 patients). Forty-two trials (41\%) reported an adequate randomisation schedule to allocate patients, 34 (33\%) trials adequately concealed treatment allocation, 35 (34\%) reported adequate blinding of patients and $20(20 \%)$ reported analyses according to the intention-to-treat principle. The average age of the patients was 47 years, most were women and of Caucasian 


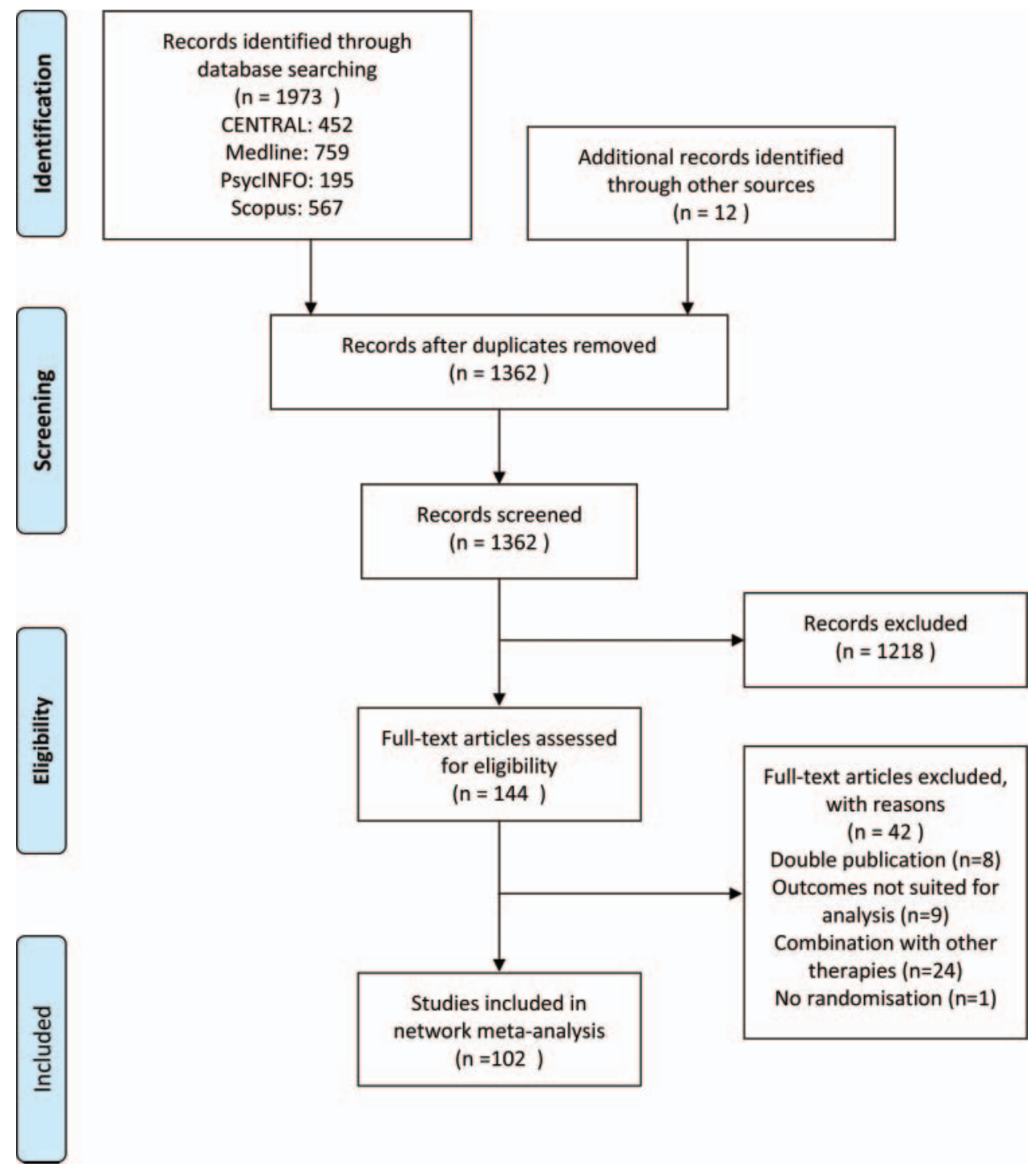

Figure 1 Flowchart for the selection of randomised trials.

origin and had an average disease duration of 8.8 years. Figure 2 shows networks of analysed comparisons for the primary outcomes.

\section{Pain}

Figure 3 (left) shows the effects on pain in 89 trials including 12979 patients between active interventions and placebo. Balneotherapy showed very large effects, SSRIs, aerobic exercise, CBT and multicomponent therapy showed moderate to large effects and TCAs, SNRIs and pregabalin showed small to moderate effects compared with placebo. For all interventions, 95\% CrIs did not overlap the null effect line. We found no clear difference between non-intervention controls and placebo (SMD 0.00, 95\% CrI -0.31 to 0.30 ) but a small difference between minimally active controls and placebo (SMD -0.22 favouring minimally active controls, $95 \%$ CrI -0.54 to 0.09 ). Heterogeneity was high with an estimate of between-trial variance $\tau^{2}$ of 0.10 (95\% CrI 0.06 to 0.17$)$. Results of analyses stratified according to methodological and clinical characteristics of included trials are shown in supplementary online table S2. Heterogeneity between trials was much reduced when restricted to trials with adequate randomisation, allocation concealment or patient blinding and large trials with at least 100 patients per arm, but there was no clear pattern for effects depending on trial characteristics and tests for interaction between effects and characteristics were all non-significant $(p \geq 0.54)$.

The scatter of effect estimates and the prediction lines observed in funnel plots for pain indicated asymmetry for both pharmacological and non-pharmacological interventions, with higher asymmetry for non-pharmacological interventions (figure 4, upper panel). Figure 3 (middle and right) shows results from network meta-analyses restricted to trials with $\geq 25$ patients per group (53 trials, 11746 patients), $\geq 50$ patients per group (25 trials, 9784 patients) and $\geq 100$ patients per group (14 trials, 8534 patients), respectively. In general, effects compared with placebo became less pronounced with increasing sample size of trials. The difference between balneotherapy and placebo was reduced in trials with $\geq 25$ patients only (SMD $-0.92,95 \%$ CrI -1.60 to -0.26 ) and no trials with $\geq 50$ patients were available for this intervention. When the network was restricted to trials with $\geq 50$ patients per group the effects of TCAs (SMD -0.32, 95\% CrI -0.66 to 0.02 ), aerobic exercise (SMD $-0.37,95 \% \mathrm{CrI}-0.72$ to -0.03 ) and CBT (SMD -0.32, 95\% CrI -0.52 to -0.11 ) compared with placebo were reduced to small to moderate differences. The effects of pregabalin and 
Table 1 Characteristics of included randomised trials

\begin{tabular}{|c|c|}
\hline Characteristic & $\begin{array}{l}\text { Total } \\
(n=102)\end{array}$ \\
\hline Number of patients randomised, median (range) & $69(14-1196)$ \\
\hline Number of multiarm trials, $\mathrm{n}(\%)$ & $28(28 \%)$ \\
\hline Year of publication, median (range) & 2005 (1986-2011 \\
\hline Mean duration of treatment, weeks, median (range) & $12(1-27)$ \\
\hline \multicolumn{2}{|l|}{ Intervention evaluated (no. of trials/no. of randomised patients) } \\
\hline Tricyclic antidepressants & $15 / 1026$ \\
\hline Selective serotonin reuptake inhibitors & $10 / 644$ \\
\hline Serotonin noradrenaline reuptake inhibitors & $10 / 5980$ \\
\hline Pregabalin & $4 / 2625$ \\
\hline Aerobic exercise & $33 / 2266$ \\
\hline Balneotherapy & $9 / 387$ \\
\hline Cognitive behavioural therapy & 20/1712 \\
\hline Multicomponent therapy & 15/1751 \\
\hline Minimally active control & $23 / 2129$ \\
\hline Placebo & $40 / 10545$ \\
\hline Non-intervention control & $38 / 2689$ \\
\hline \multicolumn{2}{|l|}{ Outcome analysed (no. of trials $/ \mathrm{no}$. of analysed patients) } \\
\hline Pain & $89 / 12979$ \\
\hline Quality of life & $78 / 12283$ \\
\hline Fatigue & $52 / 10145$ \\
\hline Sleep & $35 / 4298$ \\
\hline Dropouts & 102/14982 \\
\hline \multicolumn{2}{|l|}{ Methodological characteristics, no of trials $(\%)$} \\
\hline Adequate generation of random sequence & $42(41 \%)$ \\
\hline Adequate allocation concealment & $34(33 \%)$ \\
\hline Adequate blinding of patients & $35(34 \%)$ \\
\hline Intention-to-treat analysis performed & $20(20 \%)$ \\
\hline \multicolumn{2}{|l|}{ Characteristics of patients } \\
\hline $\begin{array}{l}\text { Patients with inflammatory rheumatic disorders excluded, } \\
\text { no of trials }(\%)\end{array}$ & $69(68 \%)$ \\
\hline Patients with mental disorders excluded, no. of trials (\%) & $48(47 \%)$ \\
\hline Percentage of Caucasians, median (range) & $100(65-100)$ \\
\hline Percentage of females, median (range) & $98(63-100)$ \\
\hline Average age, years, median (range) & $47(16-59)$ \\
\hline Average disease duration, years, median (range) & $8.8(1.3-21.6)$ \\
\hline
\end{tabular}

Thirty-eight trials did not report the percentage of Caucasians, three trials did not report the percentage of women, five trials did not report the average age of included patients and 30 trials did not report the average disease duration.

multicomponent therapy were unaffected by the restriction. Heterogeneity between trials decreased from $\tau^{2}=0.10$ in the overall analysis to $\tau^{2}=0.01$ in the analysis restricted to $\geq 50$ patients per group and, accordingly, the precision of estimates increased. Only SNRIs, pregabalin and CBT were evaluated in large trials with $\geq 100$ patients per group and showed small to moderate effects compared with placebo. Heterogeneity was low with $\tau^{2}$ of 0.00 (95\% CrI 0.00 to 0.03 ).

\section{Quality of life}

Funnel plots for quality of life indicated asymmetry for both pharmacological and non-pharmacological interventions, again with higher asymmetry for non-pharmacological interventions (figure 4, bottom). Figure 5 shows results from the network meta-analysis overall (78 trials, 12283 patients) and after stepwise restriction to trials with $\geq 25$ patients per group (55 trials, 11491 patients), $\geq 50$ patients per group (26 trials, 9415 patients) and $\geq 100$ patients per group (14 trials, 8120 patients). In the overall network, moderate to large effects for quality of life were found for all non-pharmacological interventions whereas pharmacological interventions showed small to moderate effects compared with placebo. Again we found no clear difference between non-intervention controls and placebo (SMD $-0.13,95 \%$ CrI -0.54 to 0.28 ) and a small to moderate difference between minimally active controls and placebo (SMD $-0.34,95 \%$ CrI -0.75 to 0.06 ). Heterogeneity between trials was high with a $\tau^{2}$ estimate of 0.14 (95\% CrI 0.08 to 0.23). Results of stratified analyses were similar to those observed for pain except for a significant interaction between treatment effect and intention-to-treat analysis $(p=0.016$, see supplementary online table S3).

In general, the effects became smaller with restrictions to increasing sample size of trials and between-trial heterogeneity decreased from $\tau^{2}=0.14$ in the overall analysis to $\tau^{2}=0.00$ in the analysis restricted to $\geq 50$ patients and $\geq 100$ patients per group, respectively. We found moderate effects for multicomponent therapy (SMD $-0.56,95 \% \mathrm{CrI}-0.76$ to -0.36 ) after restriction to trials with $\geq 50$ patients per group, moderate effects for CBT (SMD -0.60, 95\% CrI -0.91 to -0.29) and small effects for SNRIs (SMD $-0.21,95 \%$ CrI -0.29 to -0.14 ) and pregabalin (SMD $-0.21,95 \%$ CrI -0.34 to -0.07 ) after restriction to trials with $\geq 100$ patients per group. All other interventions were not significantly different from placebo with $95 \% \mathrm{CrI}$ overlapping the null effect.

The results of network meta-analyses of secondary outcomes, sensitivity analyses, model fit and network consistency are shown in supplementary online tables S4-S10.

\section{DISCUSSION}

In this systematic review and network meta-analysis of pharmacological and non-pharmacological interventions including 102 trials in 14982 patients with FMS, most of the trials were very small and hampered by low methodological quality. We found evidence for small-study effects and high heterogeneity and inconsistency introduced by small trials. In network meta-analyses restricted to moderate or large-sized trials, we found advantages of pharmacological interventions over placebo on pain and quality of life, which were either statistically non-significant (SSRIs and TCAs) or of questionable clinical relevance despite statistical significance (SNRIs and pregabalin). Among non-pharmacological interventions, multicomponent therapy followed by aerobic exercise and CBT was most promising for reducing pain and improving quality of life. Of the non-pharmacological interventions, only CBT was evaluated in one large trial, for which it remained unclear whether allocation was adequately concealed. ${ }^{41}$ In general, treatment effects were slightly larger for pain than for quality of life.

To our knowledge, this is the first network meta-analysis to combine pharmacological and non-pharmacological interventions in FMS. We could analyse effects of pharmacological and non-pharmacological interventions reported in different trials in a single model irrespective of the control intervention used, ${ }^{42}$ which allowed us to estimate relative effects of two interventions that have not been compared directly. This single model assumes that relative treatment effects comparing two interventions in different trials originate from a common distribution. This, however, only holds in our networks restricted to medium-sized and large trials where heterogeneity and inconsistency were low.

Our study is based on extensive literature searches and we consider it unlikely that we have missed important trials. Nevertheless, for some interventions we identified only small trials of questionable methodological quality. Stepwise restriction to trials with larger sample size led to decreases of between-trial heterogeneity and network inconsistency and, 


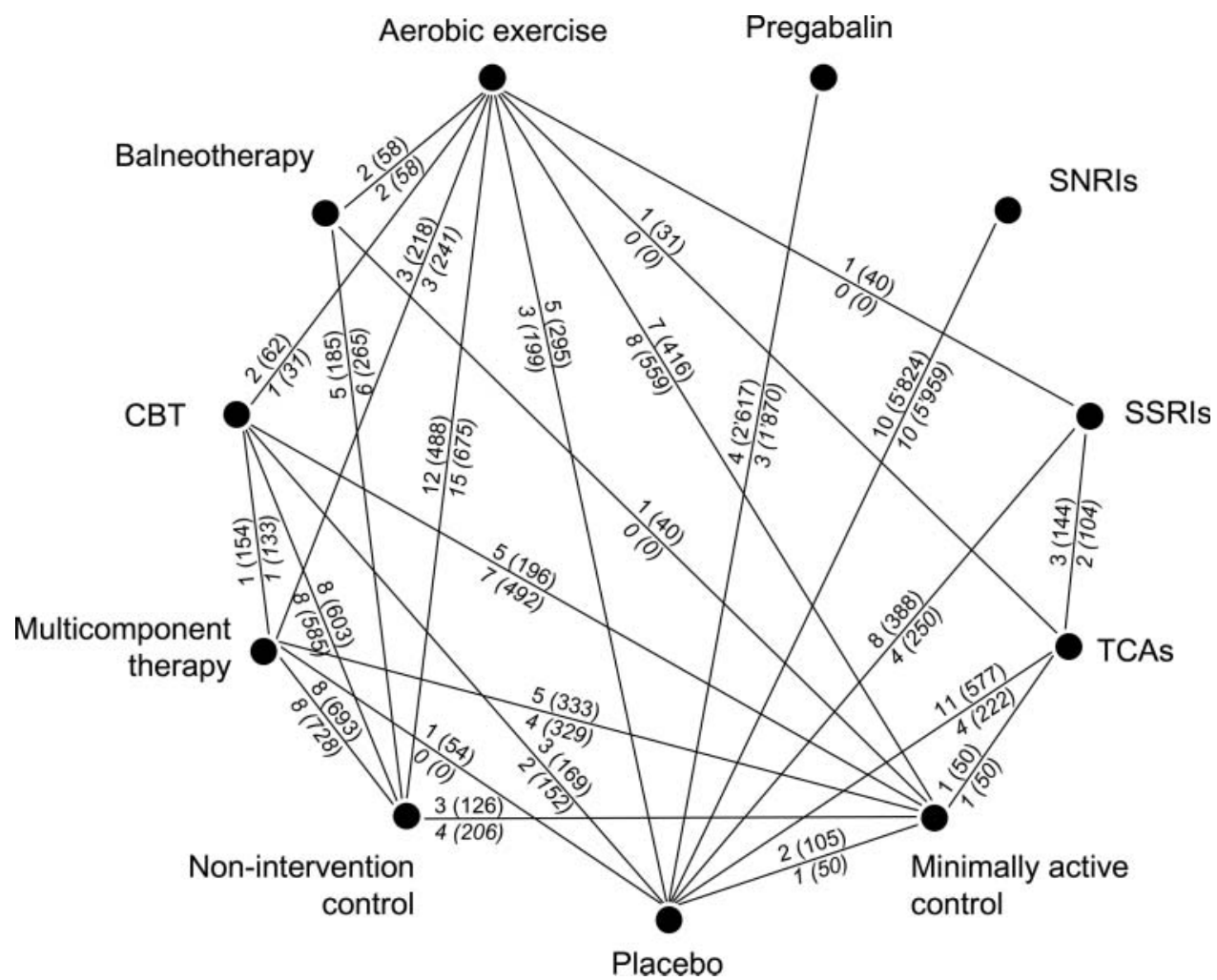

Figure 2 Network of analysed comparisons in the network meta-analyses of the primary outcomes. Numbers denote numbers of trials (number of analysed patients) per comparison for pain (normal type) and quality of life (italics). CBT, cognitive behavioural therapy; SNRIs, serotonin noradrenaline reuptake inhibitors; SSRIs, selective serotonin reuptake inhibitors; TCAs, tricyclic antidepressants.

generally, to a decrease in estimated benefits of interventions compared with placebo. We consider the results of network meta-analyses restricted to moderately-sized ( $\geq 50$ patients per trial arm) or large trials ( $\geq 100$ patients per arm) more credible than the overall analysis. However, the number of interventions available in the network decreased and, of the nonpharmacological interventions, only CBT remained in the network of large trials, which we consider one of the most important limitations. We deem estimates of CBT, balneotherapy and SSRIs most problematic as benefits changed by factors

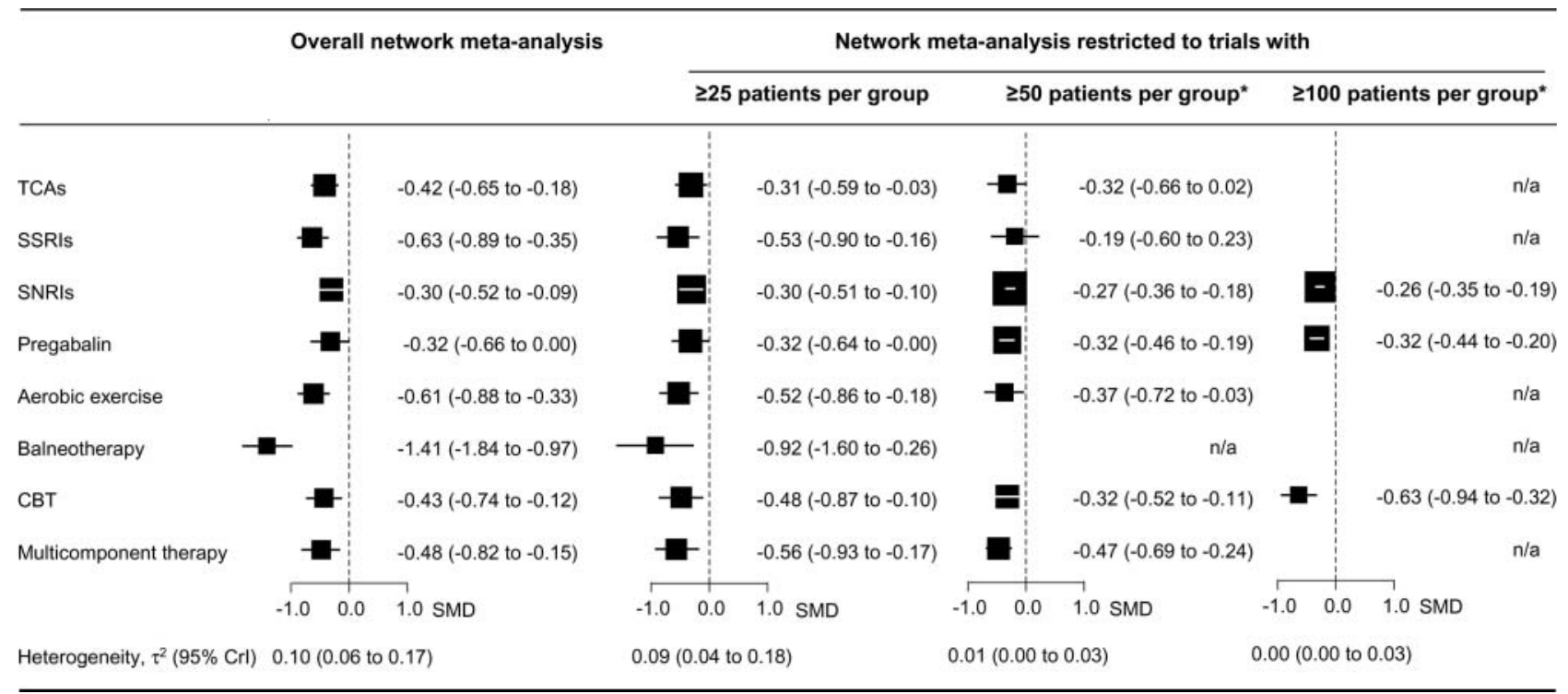

Figure 3 Estimates of standardised mean differences (SMDs) with $95 \%$ credibility intervals (95\% Crl) in pain for therapeutic interventions compared with placebo from overall network meta-analyses and network meta-analyses restricted to trials with $\geq 25, \geq 50$ and $\geq 100$ patients per group and corresponding between-trial heterogeneity variance estimates $\tau^{2}(95 \% \mathrm{Crl})$. Negative SMDs indicate benefit of therapeutic interventions compared with placebo. *Non-intervention control and placebo groups were combined to connect networks after correction for the SMD between non-intervention control and placebo of 0.00 . CBT, cognitive behavioural therapy; SNRIs, serotonin noradrenaline reuptake inhibitors; SSRIs, selective serotonin reuptake inhibitors; TCAs, tricyclic antidepressants. 


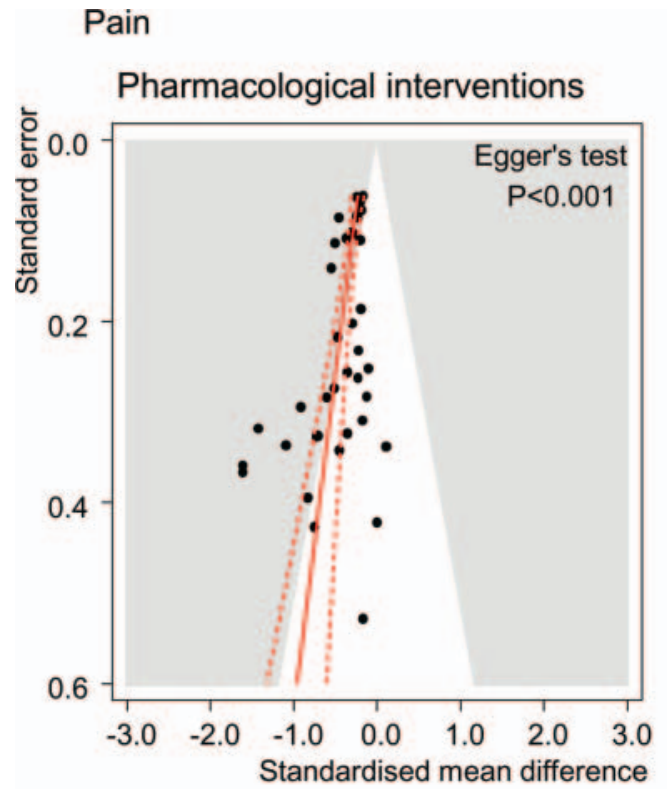

\section{Non-pharmacological interventions}

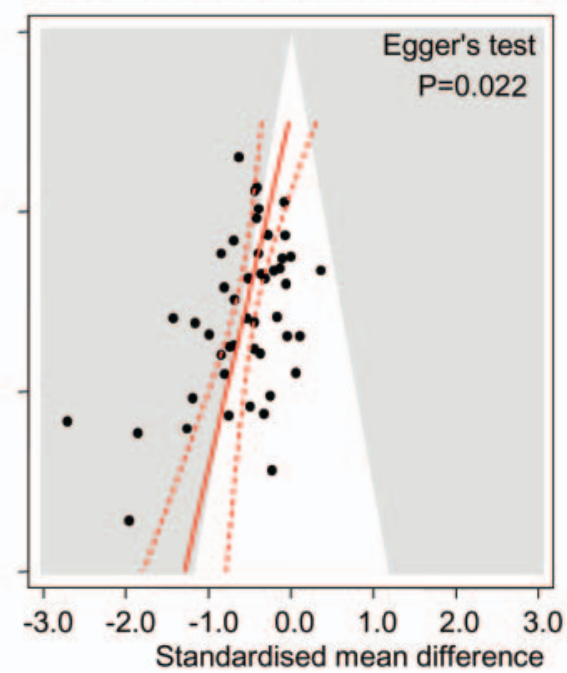

\section{Quality of life}

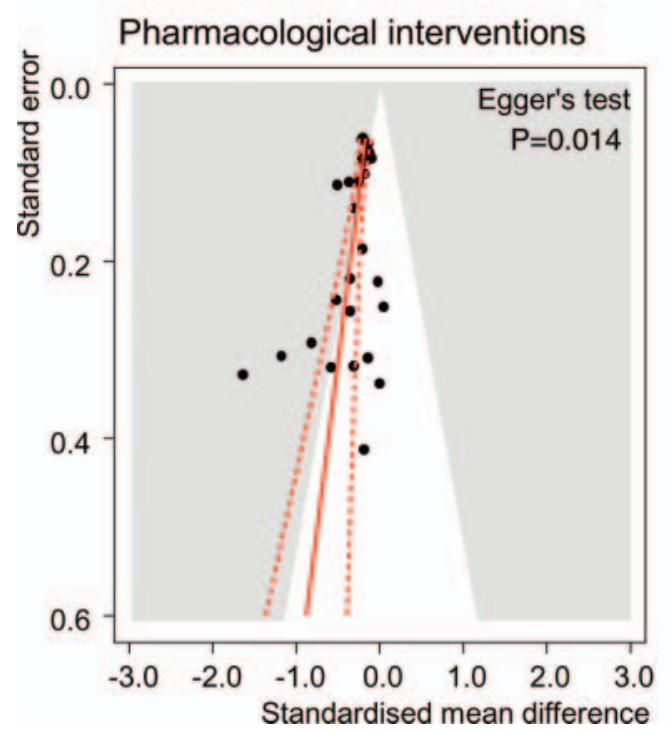

\section{Non-pharmacological interventions}

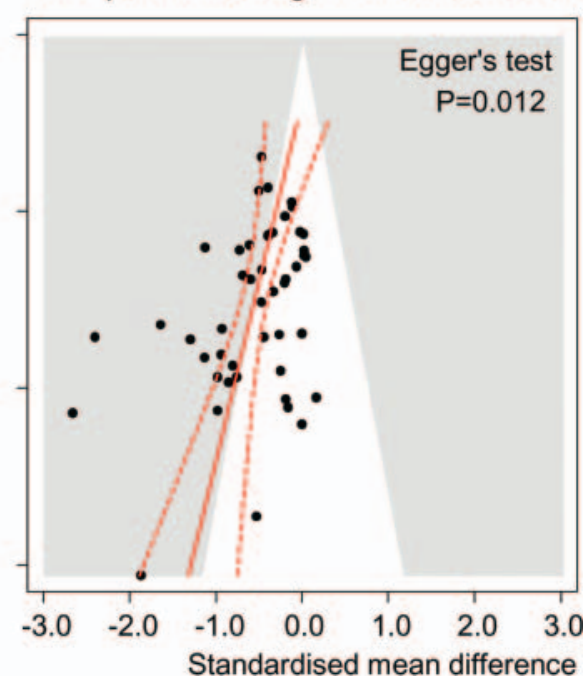

Figure 4 Contour-enhanced funnels plots for pain (top) and quality of life (bottom) comparing pharmacological (left) and non-pharmacological (right) with non-intervention control or placebo. Numbers on the $x$-axis are standardised mean differences (SMDs) and numbers on the $y$-axis are standard errors of SMDs. Areas of statistical significance at $p=0.05$ are shown in grey and prediction lines from univariable meta-regression models with the standard error as explanatory variable are shown in red. $p$ Values are derived from regression tests for asymmetry.

of 2-3 after restrictions of networks to larger trials. Since a moderate benefit of CBT was estimated in a single large trial with unclear allocation concealment, ${ }^{41}$ it is unclear whether this benefit can be confirmed in future adequately powered trials with appropriate design and conduct. Another limitation of our study is that some interventions, although widely used for the treatment of FMS in clinical practice (eg, tramadol), were not included in our analysis because they were studied in fewer than four trials.

The presence of small-study effects with large residual between-trial heterogeneity after stratification according to trial characteristics resulted in low power of interaction tests and non-significant $p$ values, and should be interpreted with caution. Conversely, statistical and visual examination of funnel plots indicated asymmetry suggestive of small-study effects. ${ }^{15}$ Although true clinical heterogeneity may explain this asymmetry, ${ }^{15} 43$ it is more plausible that asymmetry arises from the cumulative effect of various biases at the level of design, conduct, analysis and publication. As previously suggested in the field of osteoarthritis, ${ }^{14}$ sample size could therefore be viewed as a proxy for the cumulative effect of various biases prevalent in randomised FMS trials.

In clinical practice, different effectiveness on key symptoms of FMS other than pain and quality of life might lead to the selection of a combination of various treatment modalities, both pharmacological and non-pharmacological. However, we did not find any intervention for which there was conclusive evidence for clinically relevant benefits on our secondary outcomes of fatigue, sleep disturbance or acceptability. Limited data are available from randomised trials about other clinical 


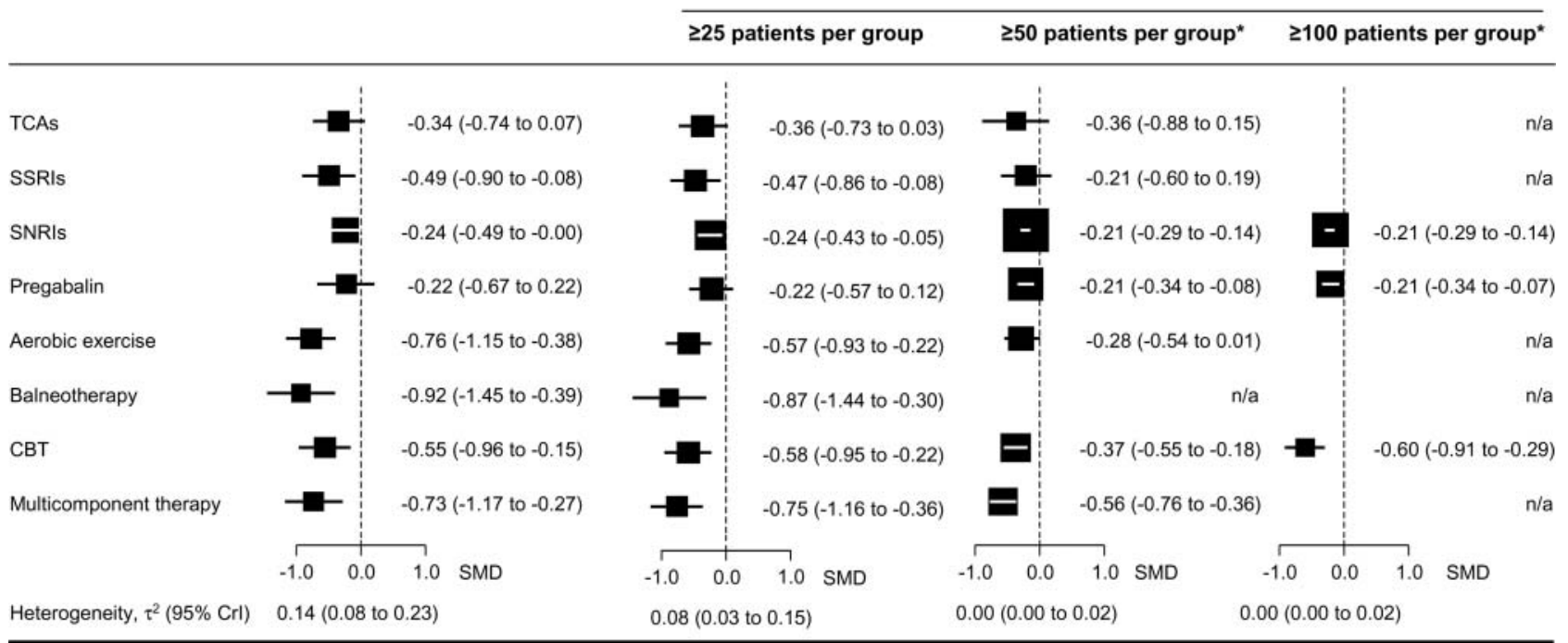

Figure 5 Estimates of standardised mean differences (SMDs) with $95 \%$ credibility intervals (95\% Crl) in quality of life for therapeutic interventions compared with placebo from overall network meta-analyses and network meta-analyses restricted to trials with $\geq 25, \geq 50$ and $\geq 100$ patients per group and corresponding between-trial heterogeneity variance estimates $\tau^{2}(95 \%$ Crl). Negative SMDs indicate benefit of therapeutic interventions compared with placebo. *Non-intervention control and placebo groups were combined to connect networks after correction for the SMD between non-intervention control and placebo of -0.13 . CBT, cognitive behavioural therapy; SNRIs, serotonin noradrenaline reuptake inhibitors; SSRIs, selective serotonin reuptake inhibitors; TCAs, tricyclic antidepressants.

dimensions such as coping or patients' beliefs, and we cannot make any conclusions about these. The generalisability of our findings derived from randomised trials to the clinical FMS population is limited, especially by the participation of selected FMS patients in randomised trials. ${ }^{16-21} 44$ Generalisability may also be hampered by protocol mandated repetitive follow-ups with extensive assessments and completion of multiple questionnaires. This would not be part of standard management and may themselves be considered as management strategies.

A recently published network meta-analysis of licensed dosages of pregabalin and the SNRIs duloxetine and milnacipran found similar benefits of these drugs over placebo on pain and quality of life, with evidence comparably robust to our network meta-analyses of large trials. ${ }^{45}$ Although statistically significant, differences in pain between pregabalin and placebo were approximately $0.6 \mathrm{~cm}$ on a $10 \mathrm{~cm}$ visual analogue scale corresponding to an SMD of $0.25 .{ }^{45}$ We consider the small advantages of pregabalin and SNRIs over placebo of questionable clinical relevance. ${ }^{40}$ Our results do not necessarily support EULAR recommendations currently favouring drug therapy over other treatment options. ${ }^{8}$ In our analysis restricted to moderate and large-sized trials, we found potentially important benefits of aerobic exercise, CBT and multicomponent therapy in the management of FMS, which is in line with recommendations of the American Pain Society and the Arbeitsgemeinschaft der Wissenschaftlichen Medizinischen Fachgesellschaften in Germany. $^{4}$

In conclusion, evidence about effective and clinically relevant treatments for FMS is limited. Additional large-scale randomised trials of high methodological quality of promising nonpharmacological interventions such as CBT, aerobic exercise and multicomponent therapy are warranted. In view of the currently available evidence, a combination of pregabalin or SNRIs as pharmacological interventions and multicomponent therapy, aerobic exercise or CBT as non-pharmacological interventions seems most promising.
Contributors All authors had full access to all the data in the study and take responsibility for the integrity of the data and the accuracy of the data analysis. EN and WH contributed equally to the study. EN, WH and PJ contributed to the study concept and design and drafted the manuscript. WH and KB contributed to data collection. EN contributed to preparation and analysis of the data. All the authors contributed to the interpretation of the data and critically reviewed the manuscript for publication.

Funding EN was a recipient of a Marie Curie Intra-European Fellowship for Career Development (grant No FP7-PEOPLE-2010-IEF-273673). WH received honoraria for one educational lecture from Janssen-Cilag in the previous 3 years. KB was supported by the Rut- and Klaus-Bahlsen Foundation in Hannover, Germany. JB and PJ received a grant (No. 105314-118312/1) from the Swiss National Science Foundation. The funding bodies had no role in the design or conduct of the study; collection, management, analysis, or interpretation of the data; or preparation, review, or approval of the manuscript, or the decision to submit the manuscript for publication.

Provenance and peer review Not commissioned; externally peer reviewed.

\section{REFERENCES}

1. Hauser W, Zimmer C, Felde E, et al. [What are the key symptoms of fibromyalgia? Results of a survey of the German Fibromyalgia Association]. Schmerz 2008;22:176-83.

2. Wolfe F, Clauw DJ, Fitzcharles MA, et al. The American College of Rheumatology preliminary diagnostic criteria for fibromyalgia and measurement of symptom severity. Arthritis Care Res (Hoboken) 2010;62:600-10.

3. Winkelmann A, Perrot S, Schaefer C, et al. Impact of fibromyalgia severity on health economic costs: results from a European cross-sectional study. Appl Health Econ Health Policy 2011;9:125-36.

4. Hauser W, Eich W, Herrmann M, et al. Fibromyalgia syndrome: classification, diagnosis, and treatment. Dtsch Arztebl Int 2009;106:383-91.

5. Branco JC, Bannwarth B, Failde I, et al. Prevalence of fibromyalgia: a survey in five European countries. Semin Arthritis Rheum 2010;39:448-53.

6. Bennett RM, Jones J, Turk DC, et al. An internet survey of 2596 people with fibromyalgia. BMC Musculoskelet Disord 2007;8:27.

7. Burckhardt CS, Goldenberg D, Crofford L, et al. Guideline for the management of fibromyalgia syndrome. Pain in adults and children. Glenview, IL: American Pain Society, 2005.

8. Carville SF, Arendt-Nielsen S, Bliddal H, et al. EULAR evidence-based recommendations for the management of fibromyalgia syndrome. Ann Rheum Dis 2008:67:536-41.

9. Lumley T. Network meta-analysis for indirect treatment comparisons. Stat Med 2002;21:2313-24. 
10. Lu G, Ades AE. Combination of direct and indirect evidence in mixed treatment comparisons. Stat Med 2004;23:3105-24.

11. Cipriani A, Furukawa TA, Salanti G, et al. Comparative efficacy and acceptability of 12 new-generation antidepressants: a multiple-treatments meta-analysis. Lancet 2009;373:746-58.

12. Juni $\mathbf{P}$, Altman DG, Egger M. Systematic reviews in health care: assessing the quality of controlled clinical trials. BMJ 2001:323:42-6.

13. Wood L, Egger M, Gluud LL, et al. Empirical evidence of bias in treatment effect estimates in controlled trials with different interventions and outcomes: meta-epidemiological study. BMJ 2008;336:601-5.

14. Nuesch E, Trelle S, Reichenbach S, et al. Small study effects in meta-analyses of osteoarthritis trials: meta-epidemiological study. BMJ 2010;341:c3515.

15. Sterne JA, Sutton AJ, loannidis JP, et al. Recommendations for examining and interpreting funnel plot asymmetry in meta-analyses of randomised controlled trials. BMJ 2011;343:d4002.

16. Hauser W, Bernardy K, Arnold B, et al. Efficacy of multicomponent treatment in fibromyalgia syndrome: a meta-analysis of randomized controlled clinical trials. Arthritis Rheum 2009;61:216-24.

17. Hauser W, Bernardy K, Uceyler N, et al. Treatment of fibromyalgia syndrome with antidepressants: a meta-analysis. JAMA 2009;301:198-209.

18. Hauser W, Bernardy K, Uceyler N, et al. Treatment of fibromyalgia syndrome with gabapentin and pregabalin — a meta-analysis of randomized controlled trials. Pain 2009;145:69-81.

19. Hauser W, Klose P, Langhorst J, et al. Efficacy of different types of aerobic exercise in fibromyalgia syndrome: a systematic review and meta-analysis of randomised controlled trials. Arthritis Res Ther 2010;12:R79.

20. Bernardy K, Fuber N, Kollner V, et al. Efficacy of cognitive-behavioral therapies in fibromyalgia syndrome - a systematic review and metaanalysis of randomized controlled trials. J Rheumatol 2010;37:1991-2005

21. Langhorst J, Musial F, Klose P, et al. Efficacy of hydrotherapy in fibromyalgia syndrome - a meta-analysis of randomized controlled clinical trials. Rheumatology (Oxford) 2009;48:1155-9.

22. Smythe HA, Moldofsky H. Two contributions to understanding of the 'fibrositis' syndrome. Bull Rheum Dis 1977;28:928-31.

23. Wolfe F, Smythe HA, Yunus MB, et al. The American College of Rheumatology 1990 criteria for the classification of fibromyalgia. Report of the Multicenter Criteria Committee. Arthritis Rheum 1990;33:160-72.

24. Yunus M, Masi AT, Calabro JJ, et al. Primary fibromyalgia (fibrositis): clinical study of 50 patients with matched normal controls. Semin Arthritis Rheum 1981;11:151-71

25. Mease P, Arnold LM, Choy EH, et al. Fibromyalgia syndrome module at OMERACT 9: domain construct. J Rheumatol 2009;36:2318-29.

26. Hollis S, Campbell F. What is meant by intention to treat analysis? Survey of published randomised controlled trials. BMJ 1999;319:670-4.

27. Cohen J. Statistical power analysis for the behavioral sciences. 2nd edn. Hillsdale, NJ: Lawrence Earlbaum, 1988.
28. Higgins JP, Whitehead A. Borrowing strength from external trials in a meta-analysis. Stat Med 1996;15:2733-49.

29. Welton NJ, Caldwell DM, Adamopoulos E, et al. Mixed treatment comparison meta-analysis of complex interventions: psychological interventions in coronary heart disease. Am J Epidemiol 2009;169:1158-65.

30. Dias S, Welton NJ, Caldwell DM, et al. Checking consistency in mixed treatment comparison meta-analysis. Stat Med 2010;29:932-44.

31. Salanti G, Marinho V, Higgins JP. A case study of multiple-treatments in meta-analysis demonstrates that covariates should be considered. J Clin Epidemiol 2009:62:857-64.

32. Sutton AJ. Bayesian methods in meta-analysis. In: Sutton AJ, Abrams KR, Jones DR, et al, eds. Methods for meta-analysis in medical research. Chichester, UK: John Wiley \& Sons, 2000:163-90.

33. Sterne JA, Egger M. Funnel plots for detecting bias in meta-analysis: guidelines on choice of axis. J Clin Epidemiol 2001;54:1046-55.

34. Palmer TM, Peters JL, Sutton AJ, et al. Contour-enhanced funnel plots for meta-analysis. Stata J 2008;8:242-54.

35. Peters JL, Sutton AJ, Jones DR, et al. Contour-enhanced meta-analysis funnel plots help distinguish publication bias from other causes of asymmetry. J Clin Epidemiol 2008;61:991-6.

36. Moreno SG, Sutton AJ, Ades AE, et al. Assessment of regression-based methods to adjust for publication bias through a comprehensive simulation study. BMC Med Res Methodol 2009:9:2

37. Shang A, Huwiler-Muntener K, Nartey L, et al. Are the clinical effects of homoeopathy placebo effects? Comparative study of placebo-controlled trials of homoeopathy and allopathy. Lancet 2005;366:726-32.

38. Sterne JA, Gavaghan D, Egger M. Publication and related bias in meta-analysis: power of statistical tests and prevalence in the literature. J Clin Epidemiol 2000;53:1119-29.

39. Altman DG, Bland JM. Interaction revisited: the difference between two estimates. BMJ 2003;326:219

40. Wandel S, Juni P, Tendal B, et al. Effects of glucosamine, chondroitin, or placebo in patients with osteoarthritis of hip or knee: network meta-analysis. BMJ 2010;341:c4675.

41. Luciano JV, Martinez N, Penarrubia-Maria MT, et al. Effectiveness of a psychoeducational treatment program implemented in general practice for fibromyalgia patients: a randomized controlled trial. Clin J Pain 2011;27:383-91.

42. Salanti G, Higgins JP, Ades AE, et al. Evaluation of networks of randomized trials Stat Methods Med Res 2008;17:279-301.

43. Egger M, Davey Smith G, Schneider M, et al. Bias in meta-analysis detected by a simple, graphical test. BMJ 1997;315:629-34.

44. Holman AJ, Neradilek MB, Dryland DD, et al. Patient-derived determinants for participation in placebo-controlled clinical trials for fibromyalgia. Curr Pain Headache Rep 2010;14:470-6.

45. Choy E, Marshall D, Gabriel ZL, et al. A systematic review and mixed treatment comparison of the efficacy of pharmacological treatments for fibromyalgia. Semin Arthritis Rheum 2011;41:335-45. 